\title{
Device Localization in Ubiquitous Computing Environments *
}

\author{
Rui Huang, Gergely V. Záruba, and Sajal Das \\ Center for Research in Wireless Mobility and Networking (CReWMaN) \\ Computer Science and Engineering Department \\ The University of Texas at Arlington \\ 416 Yates, 300NH, Arlington, TX 76019 \\ email:rxh1725@omega.uta.edu, zaruba@uta.edu,das@cse.uta.edu
}

December 10, 2006

In the ubiquitous computing environment, devices are often connected to one another on the fly to form an infrastructure-less networks called Mobile Ad Hoc Networks (MANETs). Since MANET serves as an abstract model and concept that can be seen as a superset of diverse sub-areas such as sensor networks, mesh networks or an enabler for pervasive computing, it has attracted significant research interests in the past several years. A major advantage of MANETs over regular wired or wireless networks is in their infrastructure-less nature as they can potentially be deployed more rapidly and less expensively than infrastructure-based networks. However, the lack of an underlying explicit infrastructure also becomes a major disadvantage in adapting MANETs to a wider array of applications, since existing network algorithms and protocols are not "plug-in" solutions for such dynamic networks. New algorithms need to be, and are being designed for such fundamental network tasks as addressing, topology discovery and routing.

Location discovery is emerging as one of the more important tasks as it has been observed and shown that (semi-) accurate location information can greatly improve the performance of other MANET tasks

\footnotetext{
* - Chapter of Advances in Ubiquitous Computing: Future Paradigms and Directions
} 
such as routing, energy conservation, or maintaining network security. For instance, algorithms such as Location Aided Routing (LAR) [1], GRID [3], and GOAFR+ [5] rely on the location information to provide more stable routes during unicast route discovery. The availability of location information is also required for geocast (multicast based on geographic information [6]) algorithms such as LocationBased Multicast (LBM) [2], GeoGRID [4] and Position-Based Multicast (PBM) [7]. To minimize the power consumption, the Geographical Adaptive Fidelity(GAF) algorithm [8] uses the location information to effectively modify the network density by turning off certain nodes at particular instances. Furthermore, in [9], the authors have shown that wormhole attacks can be effectively prevented when location information is available. As more algorithms are being proposed to exploit the location information in the network, it is clear that obtaining such information efficiently and accurately becomes of greater importance.

A direct way of obtaining location information is to install Global Positioning System (GPS) receivers on each node. However, this is currently impractical as GPS receivers are still relatively expensive, power-hungry, and require clear line of sight (i.e., making indoor usage impossible) to several earth-bound satellites. In sensor networks devices are imagined as small as possible and operating on a very restricted power source, thus it may not be feasible to install GPS receivers onto all sensor nodes. Localization in MANET refers to the problem of finding the locations of those non-GPS enabled nodes based on limited information such as some known beacon (also referred to as anchor) locations and measurements such as ranging distances or angles among the neighbors. The localization problem is hard for a number of reasons:

1 Geometric limitations. To pinpoint its exact location in 2-D, a node needs to know the locations of at least three beacons together with its distance from each of these beacons. Alternatively, nodes could calculate their own location based on a distance and an (absolute) angle measurement from one beacon. Even if obtaining such measurements was possible and the measurements were exact, guaranteeing that (several) beacons surround each node is impossible as MANETs 
may be randomly deployed and that in general only a small percentage of nodes are indeed beacons. Thus, a good localization algorithm needs to take advantage of multi-hop information, i.e., estimating node locations based on other nodes' location estimates.

2 Availability of measurements. For localization algorithms that require distance or angle measurements, certain sensory devices will need to be available to provide such readings. However, it is likely that not all nodes have the same sensory capacity. In other words, there is a need for the localization algorithm to work in a heterogeneous environment with different location sensory capacities.

3 Measurement error and error propagation. Even when measurement devices are available, there is a general consensus that those measurements are prone to errors. For instance, a distance measurement based on received signal strength indication (RSSI) reading is prone to multi-path fading and far field scattering. The error can be especially high when there is a significant amount of obstacles in-between the sender and the receiver. Since most localization algorithms require measurements from nodes several hops away, the measurement error is likely to aggregate along the path and eventually completely throw off the location estimate.

Despite the difficulties listed above, there have been increasing amount research effort spent into the localization problem in the recent years. The amount of effort is well justified because localization is considered an enabling technology that needs to be resolved with the best possible outcome upon which other location-dependent technologies for MANETs can be successfully employed. Researchers have been working on problem in both hardware (i.e., improving the devices measurement accuracy) and software (i.e, improving the localization algorithm).

This chapter will cover the latest advances in this field, including the following topics:

1 The need for localization. In this section, we will establish the need for better localization techniques by surveying a number of proposed algorithms for MANETs that rely on localization. 
2 Hardware devices. In this section, we will cover the latest advances in hardware design that enables localization on the smaller devices commonly seen in the ubiquitous computing environment, including the devices that measure distance ranging, angle of arrival (AoA) and interferometric ranging.

3 Survey of localization algorithms. We will survey some of the most popular localization algorithms, including those that use connectivity information, ranging and angle information. We will study the pros and cons of each algorithm and suggest their appropriate applications in ubiquitous computing.

4 Localization theory. We will cover the theoretic basis of localization techniques. We will study the necessary and sufficient conditions for a network to be localized based on the latest results from graph theory. We will show that the localization problem in general is NP-Complete. We will also introduce the Cramer Rao Bound (CRB) that is often used to analyze the hardness of different localization scenarios.

5 Future directions. We will look into a number of promising future directions for the localization techniques.

\section{Applications of Localization}

There have been numerous algorithms proposed for MANETs that rely on localization data. In this section, we provide a brief survey of them; we divided them into four categories based on their functionalities: unicast routing, multicast routing, energy consideration, and network security.

\subsection{Unicast Routing}

Routing is a specially challenging task for MANETs because their frequent topology change implies the underlying instability of any established routes. As such, routes are needed to be frequently rediscov- 
ered, reestablished, and repaired. In general, routing (i.e., route discovery and repair) involves flooding the routing control packets throughout the network. Flooding can often be quite expensive in terms of delay and bandwidth usage it incurs, both of which can greatly affect the network performance. Thus, there is a strong incentive to design efficient routing algorithms that minimize the overhead caused by any unnecessary flooding. Unicast routing based on location information, often called geometric routing or location based routing, has shown to be a viable solution to this problem.

Location-Aided Routing (LAR) [1] protocol is the first MANET routing algorithm proposed that uses location data. In LAR, every node is assumed to know its own location, and each individual location is then broadcast throughout the network. Thus, at any time $t$, every node knows the locations of any other nodes at some previous time $<t$. Based on this location information and an estimated velocity, a node can derive an estimated location range, called "expected zone", of a target node at the current time. Instead of flooding the entire network, the routing request packets can be directed to search for the target node only at this expected zone. Global flooding is performed only after the location based routing request has failed. Limiting route discovery to a smaller expected zone with LAR reduces the number of routing requests compared to the standard flooding scheme.

GRID [3] protocol uses location information as a way to form geographical clusters within the network. Based on node locations and their residency within a pre-determined grid system, nodes within the same grid block are grouped into a cluster. A cluster head (or "gateway" in [3]) is then selected for each grid block. The cluster head is responsible for servicing the routing packets. Furthermore, the cluster head can monitor the status of existing routes and reroute packets as deemed necessary. Since the cluster formation effectively simplifies the network topology, the routing overhead is reduced. A critical requirement of forming such geographical-based clusters is the availability of node location information.

In [5], the authors provided some theoretical bound to the geometric routing problem and proposed an algorithm called GOAFR+. Assuming that node locations are known using some localization technique, GOAFR+ first tries to greedily route the packet by forwarding it to the neighbor located closest to the 
destination. However, such greedy selection does not guarantee message delivery since the intermediate node closest to the destination might not have a route to it. In such cases, GOAFR+ explores the boundaries of the faces of a planarized network graph by employing the local right hand rule (i.e., always turn right) to escape the local minimum. This method of escaping local minima is also called "parameter routing," which is used in a number of other location based routing protocols as well.

Performance-wise, simulations performed by [1] and [3] have shown up to $50 \%$ of reduction in routing packets when using geometric routing compared to standard flooding. Since the overhead of flooding is proportional to network density, it has been observed that the amount of this performance increase becomes more significant when network density is increased. Furthermore, although the routing performance is impacted by the localization error, such impact is observed to be minimal. This indicates that in the case of routing, highly precise location data is not required. After all, location data is used by routing algorithms to give a direction that guides the routing packets; imprecise location data can still be used as long as the general direction is valid.

\subsection{Multicast Routing}

Similar to unicast routing, multicast routing can also benefit from location data. Multicast routing using geometric information is often referred to in the literature as geocast routing. The LocationBased Multicast (LBM) algorithm [2] is a multicast extension to the unicast Location-Aided Routing (LAR). Like LAR, which forwards the routing requests according to the location of the destination node, LBM forwards the requests according to the direction of the geocast region that contains all the multicast destinations. GeoGRID [4] is the multicast extension to GRID [3]. Like in GRID, location information is used by GeoGRID to identify the grid block where nodes reside. Multicast is done through the gateway node selected at each grid block. Based on the location of the source node and the geocast region, LBM and GeoGRID define a "forwarding region" that contains the intermediate nodes responsible for forwarding requests. The size and shape of the forwarding region have a direct impact 
on the overall performance. Shapes such as rectangles and cones have been proposed in [2].

While the standard shapes such as rectangles and cones work well in most cases, there are situations where viable routes exist only outside the forwarding region. For instance, a network can be partitioned into two sub-networks connected only through a narrow linkage due to some obstacles (e.g., two islands connected by a bridge). When the source and the destination are in separate partitions, a geometrically defined forwarding region is unlikely to cover the linkage. To prevent routing failure in such case, a routing zone based on Voronoi diagrams was proposed in [10], which partitions the network graph based on the proximity of the nodes. Again, the proximity information relies on localization information.

The Position-Based Multicast (PBM) protocol proposed in [7] attempts to optimize the multicast tree it generates by minimizing the overall path length and the overall bandwidth usage; two often contradictory objectives. To minimize the overall path length, PMB takes a greedy approach using location information. At each intermediate node, the packet is forwarded to a set of neighbors based on their overall distances to the multicast destinations. In particular, a set of the neighbors with the minimum overall distance to every destination is selected as the next set of forwarding nodes. To take in account of the bandwidth usage, the greedy selection also weighs in the size of the forwarding set in order to minimize that as well. PBM also uses parameter routing to deal with local minima. Both greedy routing and parameter routing employed by PBM rely on the location information.

\subsection{Power Management}

MANET is often being used as the model for sensor networks. Due to the recent emergence of interest in pervasive computing, sensor networks have been receiving significant research efforts. One of the major challenges of sensor networks is power management. Since sensors are commonly small in size and battery powered, conserving the energy would prolong their service time and thus the lifespan of the entire network. The Geographical Adaptive Fidelity (GAF) algorithm [8] is a network topology 
management algorithm with reduced energy consumption as its primary objective. The idea behind GAF is that there are often a large number of nodes that are redundant during packet routing in MANET. If the redundant nodes can be identified, they can then turn off their radio to save energy. For GAF, the identification of redundant nodes is accomplished by analyzing the relative location information among the neighboring nodes. More specifically, GAF divides the network into virtual grids such that all nodes in grid block $A$ are the neighbors of all nodes in grid block $B$. This way, all nodes within the same virtual grid block can be considered equivalent. To conserve energy during packet routing, GAF only turns on the radio for one of nodes in each grid block. The active node is periodically "roundrobinned" to achieve load-balancing. Analysis and simulations performed in [8] show that GAF can reduce overall energy consumption by $40 \%$ to $60 \%$.

\subsection{Security}

In [9], the authors proposed a technique called "packet leashes" to defend against wormhole attacks in MANETs. A wormhole attack is a type of security breach where an adversary intercepts incoming packets and tunnels them to another part of the network via a single long-range directional wireless link or through a direct wired link. From there, the adversary can retransmit the packets to the network. Note that this type of "capture-and-retransmit" attack can be immune to common packet encryption methods, since the adversary does not need to read the packet content. Wormhole attacks can severely disrupt ad hoc routing protocols such as Ad hoc On-Demand Distance Vector Routing (AODV) or Dynamic Source Routing (DSR), and cause a denial of service to the network. The core of "packet leashes" is based on two assumptions: i) all nodes know their own locations, and ii) all nodes are synchronized. To enable packet leashes, the sender node encloses its location and transmission time-stamp within the packet. At the receiver node, the packet leash is validated against the receiver's own location and clock. In particular, the sender location information gives the distance from the original sender to the receiver, and the time-stamp gives the transmission duration of the packet. Based on the transmission 
duration and signal propagation model, factored in some error tolerance, the receiver can validate the estimated distance the packet has traveled against the true distance to tell if the packet is indeed coming from the original sender or an imposer at some other location. Thus, the location information and timestamp provide a virtual leash to limit the effective range of the packet so that it cannot be exploited by wormhole attackers.

From the previous discussion on the location-dependent algorithms that encompass a wide range of domains, it is quite obvious that providing location information (i.e., localization) to MANET is becoming an increasingly important task. In fact, localization is now widely regarded as an "enabling technology" for MANET that needs to be addressed before other location-dependent techniques can be realized in the real world [11].

\section{Hardware Devices for Localization}

In this section, we study a number of hardware devices that enable localization in MANETs for ubiquitous computing environments. A Global Positioning System (GPS) [12] receiver can provide the absolute location. However, its cost, size and power requirement prevent it from being installed at every network node. Thus, those non-GPS nodes have to be localized using measurements provided by alternative hardware devices. There are five general types of measurements as follows: i) connectivity only, ii) RSSI (radio signal strength indicator) ranging, iii) ToA (time of arrival) ranging, iv) AoA (angle of arrival), and v) interferometric ranging.

\subsection{Connectivity Only Measurement}

At a minimum, a node can detect connectivity to its neighbors, i.e., its one-hop neighborhood. The connectivity only measurement is a binary reading between two nodes of either "true" or "false" indicating whether they are neighbors. Based on this connectivity information, one can derive the general 
proximity of the nodes and use it as a way to localize the network.

\subsection{RSSI Ranging Measurement}

A node can be localized using multilateration if the distances (i.e., the ranges) to three or more known locations are obtained. The distances can be obtained, e.g., by measuring RSSI or ToA. In RSSI, the receiver measures the received signal strength and compares it with the transmitted signal strength. The difference (in $\mathrm{dB}$ ) is then applied to the inverse of the signal propagation model to provide with a distance measurement. Sensors that measure RSSI are widely available to mobile devices. Indeed, most off-the-shelf technologies implicitly provide such information (e.g., most WiFi, Bluetooth and IEEE802.15.4 chipsets do). The drawback of RSSI based measurements is that they can be very inaccurate because an exact model of the propagation environment is often unavailable. Experiments in [24] have shown that when no obstacle exists between the sender and the receiver, RSSI can provide a distance estimate with an accuracy of a few meters. However, at a less than deal environment, the result is often unpredictable. Furthermore, low cost RSSI receivers are often variable in their transmission power due to the lack of calibration.

In the outdoor environment with minimum obstacles, signal propagation decay is proportional to $d^{n_{p}}$, where $d$ is the distance the signal has traveled. However, in the actual environment where obstacles exist, multipath signals and shadowing become two major sources of noises that impact the actual RSSI. In general, those noises are commonly modeled as a random process during localization. Let $P_{i, j}$ be the RSSI (in $\mathrm{dB}$ ) obtained at the receiver node $j$ from the sender node $i . P_{i, j}$ is commonly modeled as a Normal distribution [11]

$$
P_{i, j}=N\left(\bar{P}_{i, j}, \sigma_{d B}^{2}\right)
$$

where $\bar{P}_{i, j}$ is the mean power in $\mathrm{dB}$ and $\sigma_{d B}^{2}$ is the variance caused by noise factor such as shadowing. 
$\bar{P}_{i, j}$ is further defined as the power reduction from a reference location:

$$
\bar{P}_{i, j}=P_{0}-10 n_{p} \log _{10}\left(d_{i, j} / d_{0}\right)
$$

where $P_{0}$ is the power at a reference location at the distance $d_{0}$ (commonly $d_{0}=1 \mathrm{~m}$ ). $n_{p}$ is an environment-dependent path loss exponent that is assumed to be known from prior measurements (theoretically $n_{p}=2$ ). $d_{i, j}$ is the Euclidean distance between nodes $i$ and $j$.

\subsection{ToA Ranging Measurement}

Although ToA is used for radio signals in GPS, it is mostly used in the context of acoustic or ultrasonic signals in inexpensive ToA tracking (as propagation speeds are five orders of magnitude less). For instance, the Medusa node in [24] is an implementation of ToA ranging using ultrasonic signals. ToA measures the time signals travel from the sender to the receiver. The distance between nodes is obtained by multiplying this time with the signal propagation speed. In spite of the additive noise and multipath, in general distance measures based on ToA are more accurate than RSSI based measures. However, special acoustic transceivers have to be employed on each node and synchronization among the nodes needs to be established. Sensor network clock synchronization algorithms accurate to the order of $10 \mu \mathrm{s}$ have been reported [17]. As mentioned earlier, ToA may also be used together with radio signals, but current technology is not mature enough to provide with a satisfactory precision over smaller distances inexpensively.

Let $i$ be the sender node and $j$ be the receiver node, ToA measurement $T_{i, j}$ is often modeled as a Normal distribution [11]:

$$
T_{i, j}=N\left(d_{i, j} / c, \sigma_{T}^{2}\right)
$$

where $d_{i, j}$ is the Euclidean distance between $i$ and $j, c$ is the signal propagation speed, and $\sigma_{T}^{2}$ is the variance caused by noises. 


\subsection{AoA Measurement}

A node can be localized if the angles between itself and two beacons are known. Thus, it is possible to localize the network based on the angle information (i.e., bearing, or angle of arrival (AoA). Currently, there is no off-the-self device that offers AoA sensing capability. However, a number of prototype devices are available. For instance, Cricket Compass [18] is a small form device that uses ultrasonic measurements and fixed beacons to obtain acoustic signal orientations. In [19], a rotating directional antenna is attached to an $801.11 \mathrm{~b}$ base station. By measuring the maximum received signal strength, a median error of $22^{\circ}$ can be obtained from the sensor. The challenge here is to design the AoA sensing device that has small form factor and low energy consumption. In [20], the authors outline a solution with a ring of charge-coupled devices (CCDs) to measure AoA with relatively low energy consumption.

In general, AoA is also modeled as a Normal distribution. Let the true angle between the sender $i$ and $j$ be $a_{i, j}$, the AoA measurement between $i$ and $j$ is therefore

$$
A_{i, j}=N\left(a_{i, j}, \sigma_{a}^{2}\right)
$$

where $\sigma_{a}^{2}$ is the angle variance. Theoretical results for acoustic-based AoA estimation show standard deviation $\sigma_{a}$ is between $2^{\circ}$ to $6^{\circ}$, depending on range [13]. RSSI based AoA method with $\sigma_{a}$ on the order of $3^{\circ}$ has been reported in [14].

\subsection{Interferometric Ranging Measurement}

Interferometric ranging is a "widely used technique in both radio and optical astronomy to determine the precise angular position of celestial bodies as well as objects on the ground [15]." Interferometric ranging exploits the property that the relative phase offset between two receivers determines their distances to the two simultaneous senders. Due to the recent advancement in hardware, it is now possible to implement interferometric ranging sensors in much smaller form factor so that it can be used 


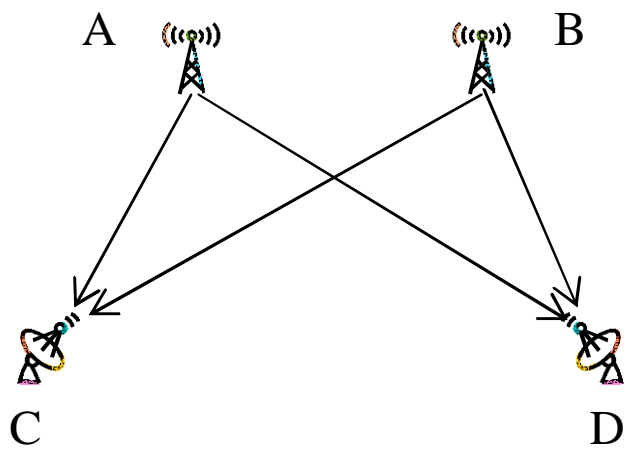

Figure 1: The interferometric ranging measurement of the q-range $d_{A B C D}=d_{A D}-d_{B D}+d_{B C}-d_{A C}$. Here, node $A$ and $B$ are the senders, and node $C$ and $D$ are the receivers.

for localization [16]. By synchronizing the transmission at the two senders, each of which sends a signal at a slightly different frequency, the receivers can derive the relative phase offset of the two signals by comparing the RSSI readings. The distance difference (also called the $q$-range) can then be calculated from the relative phase offset with high accuracy. A q-range obtained from interferometric ranging from two senders $A$ and $B$, and two receivers $C$ and $D$ is the distance difference $d_{A B C D}=d_{A D}-d_{B D}+d_{B C}-d_{A C}+e$, where $e$ is the measurement error (Figure 1).

A major advantage of interferometric ranging is that the measurement could be extremely accurate compared to noise-prone RSSI readings. In a recent experiment [16], in which 16 nodes are deployed in a $4 \times 4$ grid over a $18 \times 18$ meters flat grassy area with no obstruction, the maximum q-range error was shown to be around 0.1 meters while the medium error was less than 0.04 meters. However, interferometric ranging is more difficult to implement partially due to the following reasons:

1. The measurement can be impacted by various sources of noise such as frequency drift, ground multipath error, and time synchronization error [16]. Frequencies of the transmissions need to be precisely calibrated, as any carrier frequency drift and phase noise would directly impact the observed phase offset. Precise time synchronization is needed at the senders of a q-range. Thus, there will be overhead to maintain clock synchronization.

2. A significantly larger number of measurements are needed for localization than using direct rang- 
ing techniques. While there are also a large number of measurements available $\left(O\left(n^{4}\right)\right)$ even for a small network, only a small subset of them are independent of each other. The rest merely provide redundant information. It has been shown in [15] that the number of independent measurement using interferometric measurements is $O\left(n^{2}\right)$, which is significantly higher than with RSSI and AOA ranging $(O(n))$. Considering the localization problem in relative coordinates, for a network of $n$ nodes there are $2 n-3$ unknowns in two dimensions and $3 n-6$ unknowns in three dimensions ${ }^{1}$. Thus, the smallest network that can be localized using interferometric measurements is a fully-connected network with a population of $n=6$, where there are 9 independent measurements available to cover 9 unknowns. The large number of q-ranges available/required indicates a scalability issue for larger networks.

3. Since each measurement involves four nodes, more collaboration is required between nodes. Due to the requirement of synchronized transmission, the senders have to collaborate in scheduling their transmission. Also, the receivers have to collaborate to derive the relative phase offset. This collaboration requires sophisticated protocols to be implemented so as to reduce the communication overhead.

Those difficulties rooted in the physical characteristics of interferometric ranging devices affect the algorithmic design of the localization algorithm. As we will see in the following section, the localization algorithms based on interferometric ranging measurements tend to be more difficult to design.

\section{Localization Algorithms}

Obtaining measurements such as distance ranging and angle of arrival is only the first step of localization. To calculate the actual node location, we will have to rely on localization algorithms. While there are various ways of classifying localization algorithms, we feel it is more logical to classify them

\footnotetext{
${ }^{1}$ This is because the relative coordinates are invariant under translation, rotation and reflection. Thus, in two dimensions, we have $2 n-3$ degrees of freedom, where translation, rotation and reflection each reduce one degree of freedom.
} 
according to the measurement assumptions as follows: i) connectivity-only, ii) range-based, iii) anglebased, iv) interferometric ranging based, v) hybrid, and vi) mobility-based.

\subsection{Connectivity-Based Algorithms}

A number of localization methods rely on connectivity information only. These types of methods are also referred to as "range-free" methods in the literature. For instance, the Centroid method [21] estimates the location of an unknown node as the average of its neighboring beacon locations. To provide sufficient localization coverage, the Centroid method requires more powerful beacons with a large transmission range.

The APIT (Approximated Point-In-Triangulation) method [22] estimates the node location by isolating the area using various triangles formed by beacons. For each triangle formed by three beacons, the node is either in or out of the triangle. For instance in Figure 2(a), if it can be determined the node $G$ is inside $\triangle A B C$ and $\triangle D E F$, then its location can be isolated to the shaded overlapping area of the two triangles. To determine whether a node is inside or outside the triangle, APIT compares the RSSI readings from the beacons at the node with those at its neighbors. Intuitively, smaller RSSI reading means a shorter distance (i.e., closer to the beacon) and vice versa. If there does not exist a neighbor that is further from (or closer to) all beacons simultaneously, then the node is inside triangle with high probability. For instance in Figure 2(b), a neighbor of $D, E$, can be measured to be further away from the beacon $A, B$ and $C$ because it has smaller RSSI readings comparing to $D$. Thus, $D$ is considered as to be outside $\triangle A B C$. Conversely, if $D$ is inside $\triangle A B C$ (Figure 2(c)), then it is likely that its neighbors will be closer to (or further away from) some (but not all) of the triangle points. Clearly, this test does not guarantee correctness every time. However, since there are a large number of triangles available

for the test ( $O\left(n^{3}\right)$ for $\mathrm{n}$ beacons), error can be effectively controlled. Indeed, simulations performed in [22] indicated that APIT gives more accurate localization than the Centroid method when the beacon density is higher. Note that although APIT makes use of RSSI, it is only used to derive the relative 


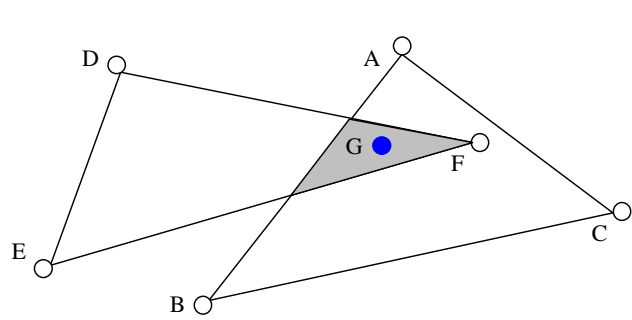

(a) localization using overlapping triangles

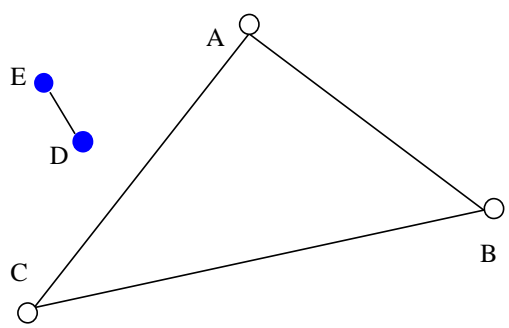

(b) node outside a triangle

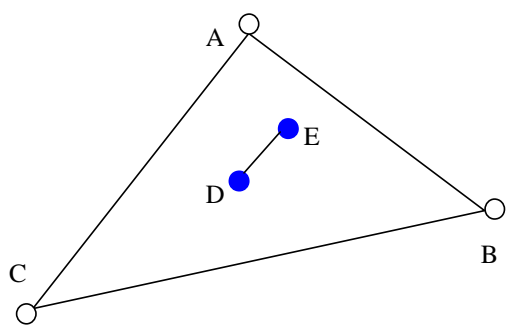

(c) node inside a triangle

Figure 2: APIT.

proximity, but not the absolute distance. Thus, we classify it as a connectivity-based algorithm.

Both the Centroid and APIT methods try to localize the node directly from the beacons 1 hop away. Thus, to provide better localization coverage, they require either a large number of beacons or a large beacon transmission range. The DV-Hop method [23] relaxes such requirement by providing a way to localize from the beacons several hops away. In DV-Hop, each beacon floods its location to the entire network much like the distance vector (DV) routing protocol. Each node maintains a DV table of the beacon locations it has heard along with the shortest hop count to them. A node will only forward the location broadcast if it has a shorter hop count than the current one in its table. In addition, when a beacon has heard the broadcast originated from another beacon, it can derive the distance-per-hop information based on the physical distance between the two beacons and the hop count accumulated along the path. The distance-per-hop information is then broadcast to other nodes. To localize, a node extracts the hop counts to the beacons from its DV table and converts them into distances using the average distance-per-hop information it has received. The node can then estimate its location using multilateration based on the distances to the beacons. For instance in Figure 3, the node $D$ can triangulate based on the location broadcast from the beacons $A, B$, and $C$ stored in its DV table. The distance-per-hop is calculated as the average of the distances per hop among all the beacons. Compared to Centroid and APIT, DV-Hop requires much less number of beacons. It does however have greater communication overhead since it requires multiple message flooding.

The above connectivity-based localization methods assume the nodes are stationary. The MCL 


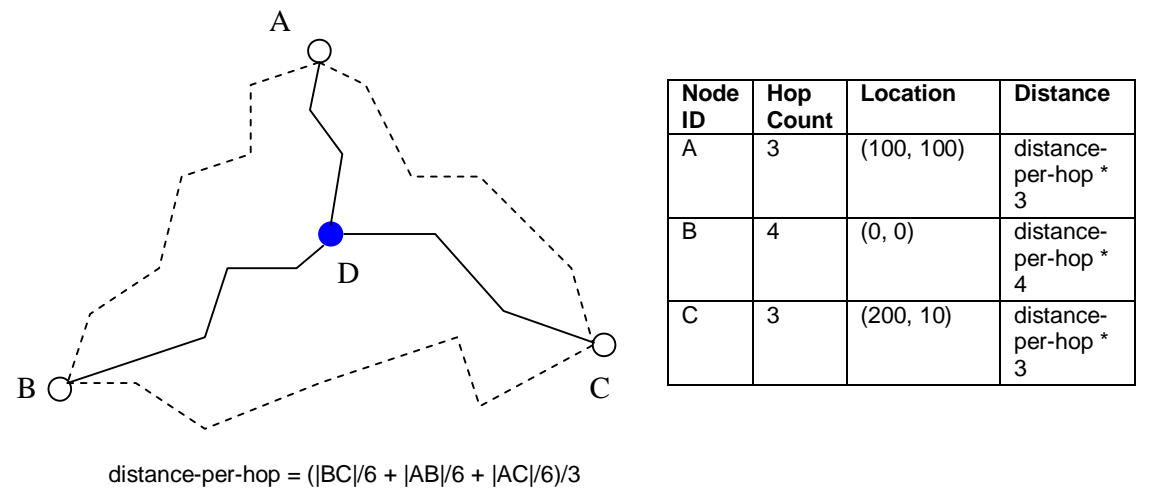

Figure 3: DV-Hop.

(Monte Carlo localization) method [27] takes a novel approach by making use of the node mobility. As a node moves, it becomes connected or disconnected to other nodes. Based on the connectivity observation, a unit-disk connectivity model, and a simple random movement model of the node, MCL updates the probability distribution of the possible node location. Simulation in [27] has reported as much as three times of localization accuracy when compared to the Centroid method.

In general, connectivity-based localization algorithms tend to be simple to implement, and they depend less on special hardware. However, due to the lack of more precise measurement, the location estimates they provide tend to be less accurate. A large number of beacons need to be deployed in order to improve their accuracy. Sparse networks by nature contain less connectivity information, and thus they are more difficult to localize accurately using connectivity-based localization methods.

\subsection{RSSI and ToA Range-Based Algorithms}

Many algorithms use the RSSI and ToA measurement to derive the distance to the senders. The DVDistance method [23] behaves much like the connectivity-based DV-Hop method. But instead of incrementing the hop count, DV-Distance increments the distance between from hop to hop as beacons broadcast their locations. Since the distance at each hop can be quite different, DV-Distance can obtain more accurate range to the beacons compared to DV-Hop, which only considers the average case. How- 


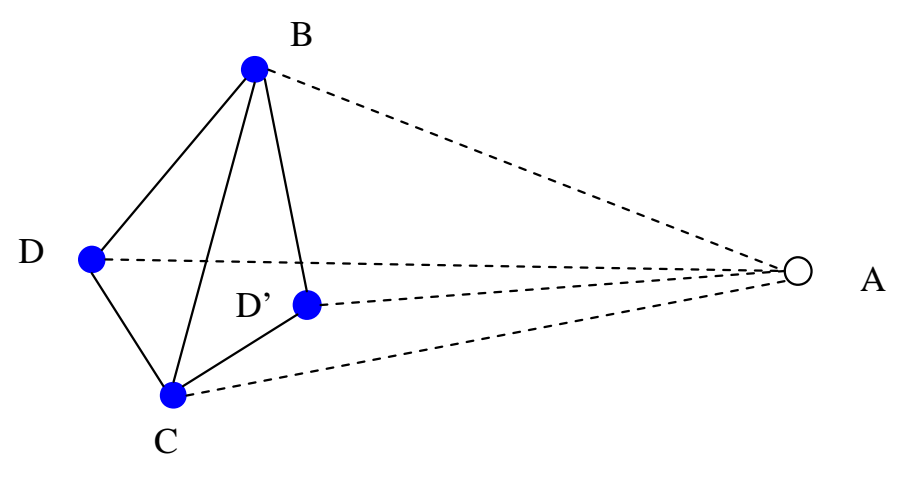

Figure 4: Multihop Distance Derivation in Euclidean.

ever, its performance becomes heavily dependent on the ranging measurement accuracy. The Euclidean method [23] tries to derive the distance to a beacon that is several hops away by measuring RSSI or ToA to its neighbors. For instance in Figure 4, the node $D$ is several hops away from the beacon $A$. To derive its distance to $A, D$ obtains the distance using RSSI or ToA to two neighbors $B$ and $C$, where the distance $A B, A C$ and $B C$ are known. The distance $A D$ is the second diagonal of the quadrilateral $A B D C$. Depending on whether $A B D C$ is convex or concave, two solutions of $A D$ exist. This ambiguity can be solved by examining multiple quadrilaterals like $A B D C$. Once the distances to at least three beacons have been obtained, both DV-Distance and Euclidean method estimate the node location using mulitlateration.

The Collaborative Multilateration method [24] is also based on mulitlateration from ranging. However, it allows nodes being triangulated from non-beacon nodes. Initially, all non-beacon nodes are assumed to be at some random locations. As a node receives its neighbors' estimated locations, it tries to triangulate its new location with the least mean square error. The newly estimated location is then sent back to the neighbors for their own mulitlateration. The process is iterated multiple times, and the idea is that eventually the location information from the beacons will propagate to remote nodes via collaborative multilateration. However, it is foreseeable that the nodes further away from the beacons would be slow to converge. The Hop-TERRAIN method [25] makes an improvement in this regard by using the DV-Hop method to derive an initial coarse location. It then runs the collaborative multi- 


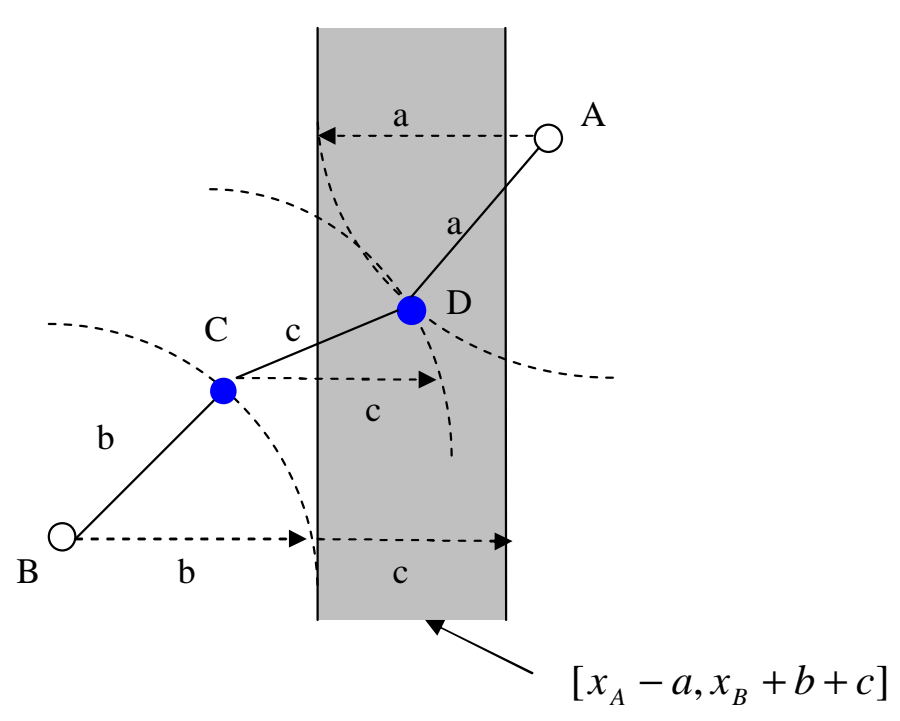

Figure 5: Bounded Region in n-Hop Multilateration.

lateration to further refine the localization results from the distance and location information from the neighbors. The n-Hop Multilateration method proposed in [28] uses a bounded box model instead of DV-Hop to provide initial location estimates. For instance in Figure 5, while node $D$ is two hops away from the beacon $B$ and one hop away from the beacon $A$, it is still bounded by the distance constraints. The bound on the $x$ coordinates is $\left[x_{A}-a, x_{B}+b+c\right]$, where $a=|A D|, b=|B C|$ and $c=\mid C D$. Using this kind of geometric bounding via multihops, an initial location of the node can be derived.

The iterative multilateration provides a way to deal with the difficult question of how to effectively use the beacon information several hops away. However, since it treats location estimates from nonbeacons the same as beacons, the beacon information can be quickly watered down by the inaccuracy of non-beacons. The probabilistic localization method in [26] explicitly considers the location uncertainty of non-beacons by the means of probability distributions. In particular, each node location is not represented by a singular value but a probability distribution in terms of particles. Initially, all nonbeacons have a uniformly distributed particle distributions. To localize, nodes exchange their particle distributions among the neighbors and run Monte-Carlo filtering based on the RSSI or ToA measurement data to update the particles. Eventually, the particles will be refined to the true location of where 
the node resides. The particle filtering method allows collaborative localization as shown in Figure 6. Here, nodes 2, 3, and 4 are beacon nodes, while nodes 0 and 1 are non-beacons. Of the beacons, node 0 can receive signals only from nodes 1 and 4 , and node 1 can receive signals from only nodes 0,2 , and 3. From the signal strength readings, non-beacons estimate their distances to their neighbors. The probability distribution of the estimated location is represented by the particles (dots) in the graph. In sub-figure (a), where node 1 is removed, node 0 can only receive signals from node 4 ; thus as the particle distribution indicates, the probability distribution where node 0 is most likely located concentrates on a circle around node 4 . In sub-figure (b), where node 0 is removed, node 1 can receive signals from nodes 2 and 3; thus the most likely locations for node 1 center around two areas where "transmission circles" around node 2 and 3 intersect. Intuitively, in order to localize itself, a node needs to receive location information from a minimum of three beacons either directly or indirectly. In both case (a) and case (b), the exact location of the nodes 0 and 1 cannot be deduced because they do not receive location information from all three beacons. In (c) and (d), where all nodes are available, nodes 0 and 1 are able to communicate to each other and exchange their particle distributions. Thus, their probability densities will represent their actual locations much closer even though neither node receives location information from all three beacons directly.

Compared to range-free methods, range-based methods give more accurate location estimates when ranging data is reliable. However, depending on the deployment environment, ranging techniques based on RSSI tend to be error-prone and strong filtering is required. The ranging error could ultimately destroy the localization accuracy if it is allowed to propagate through the network unbounded. Furthermore, different methods generally exploit the trade-off between the estimation accuracy and the estimation coverage. For instance, given the same network scenario, the Euclidean method is capable of generating more accurate location estimates of a smaller subset of nodes, whereas the DV-Hop method has better coverage but worse accuracy. Regardless of the tradeoff, a common characteristic shared by distance-based localization algorithms is that they require a relatively high network density in order to achieve better results. Based on the extensive simulation of DV-Distance, Euclidean and multilatera- 


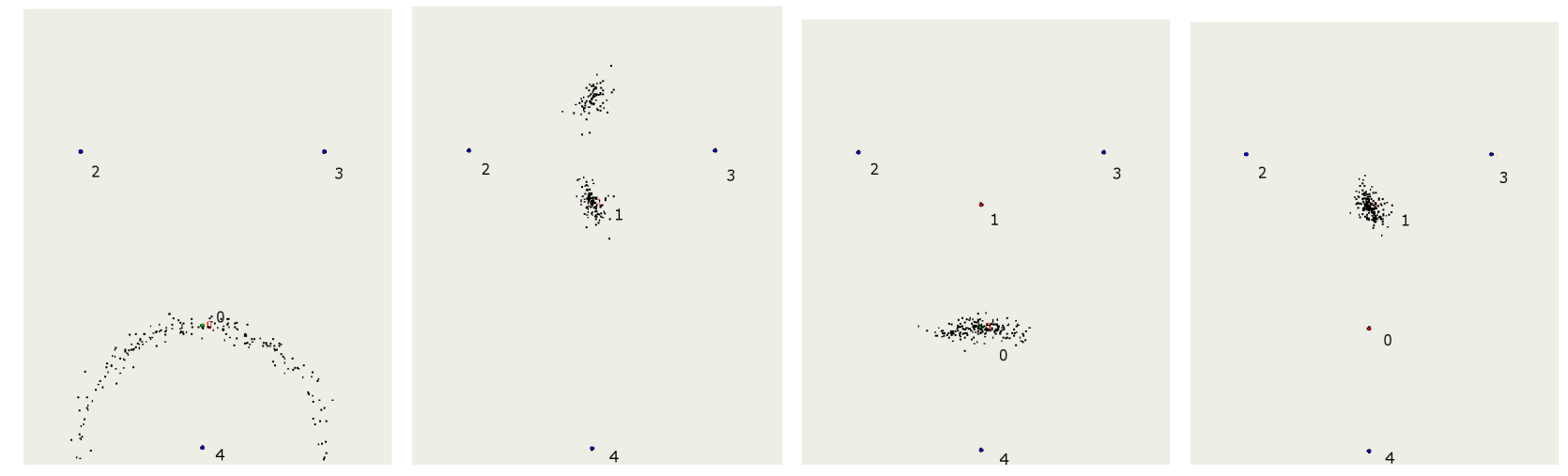

(a) Particle distribution of (b) Particle distribution of (c) Particle distribution of (d) Particle distribution of node 0 when node 1 is not node 1 when node 0 is not node 0 when node 1 is node 1 when node 0 is present present present present

Figure 6: Collaborative Localization Using Particle Filters.

tion methods performed in [20], it can be concluded that those distance-based localization algorithms "require an average degree of 11-12 nodes within the ranging neighborhood in order to achieve $90 \%$ localization coverage with $5 \%$ accuracy [20]."

\subsection{Angle-Based Algorithms}

Even though the future of AoA sensing devices is still unclear, some works have been published on localization using angle information. Simulation studies in [20] also show that when AoA of the signals is used in addition to the distance measurement, the localization accuracy and coverage can be drastically improved. This should not come as a surprising conclusion, as nodes need to communicate with only one neighbor to perform localization if they can obtain both AoA and distance measurements. The work in [20] also presents three variations of a weighted mean square error algorithm that localizes the nodes, each of which is designed to work with one of the three measurement types: i) distance-only measure, ii) distance plus a more accurate AoA measure (up to $8^{\circ}$ of precision) and iii) distance plus a less accurate AoA measure (up to $60^{\circ}$ of precision). The less accurate AoA measurement method is sometimes referred to as sectoring. Simulations in [20] show that the localization accuracy and 


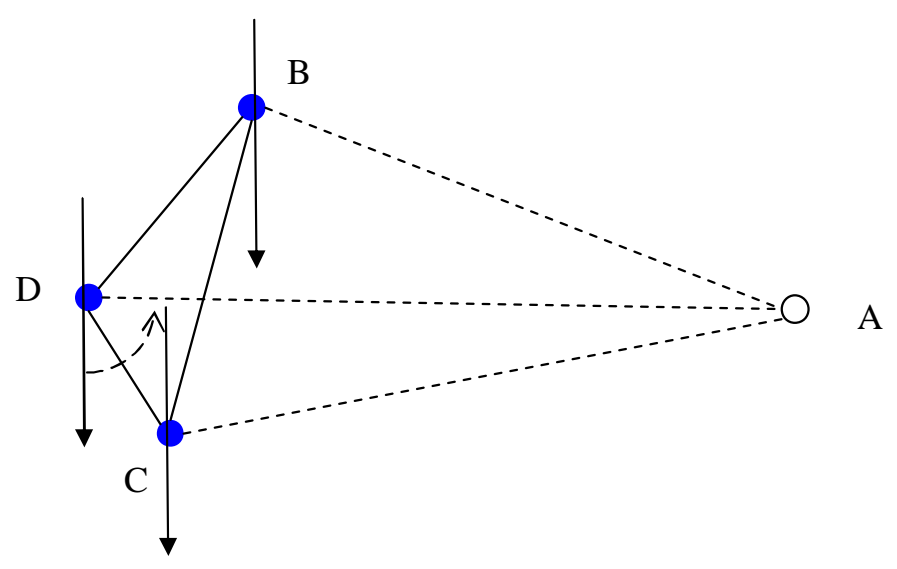

Figure 7: Multihop Distance Derivation in AoA Triangulation.

coverage can be greatly improved with such coarse sectoring measurement as well.

In order to localize with only AoA measurement, the AoA triangulation method proposed in [29] can be used. The triangulation takes several AoA measurements from beacons and estimates the node location with least square error. To propagate the AoA measurement for more than one hop, the AoA triangulation method uses a approach called orientation forwarding that is similar to the Euclidean method for distance ranging. For instance in Figure 7, let AoA measurement be the bearing against South. For node $D$ to derive its bearing to the beacon $A$ (i.e., $\angle S_{D} D A$ ), it can contact two neighbors, $B$ and $C$, with known AoA measurements from the beacon $A$ (i.e., $\angle S_{B} B A$ and $\angle S_{C} C A$ are known). Furthermore, $B, C$ and $D$ can measure the AoA of each other to give the readings of $\angle S_{B} B C, \angle S_{B} B D$, $\angle S_{C} C B, \angle S_{C} C D, \angle S_{D} D B$ and $\angle S_{D} D C$. From there, all angles in $\triangle A B C$ and $\triangle B C D$ can be determined. The bearing from $A$ to $D$ can be derived as $\angle S_{D} D A=\angle S_{D} D C+\angle C D A$, where $\angle S_{D} D C$ is known, and $\angle C D A$ can be determined from $\triangle A B C$ and $\triangle B C D$. Using the orientation forwarding method, the bearing to beacons can be propagated through mutlitops, which can then be used to triangulate the location. However, much like the case of distance propagation, measurement error becomes aggregated at each hop. Simulations in [29] have reported a near linear error increase to the hop count.

Overall, due to the limited availability of AoA sensing devices, relatively few algorithms have been proposed for AoA. However, it is conceivable that some localization algorithms originally proposed for 
RSSI or ToA ranging can be adapted to AoA. For instance, the probabilistic algorithm in [26] can be updated to accept AoA measurements by simply providing an alternative measurement model for AoA during particle filtering.

\subsection{Interferometric-Based Algorithms}

Due to the fact that interferometric sensing devices for localization are relatively new, there have been only a limited number of localization algorithms proposed for this type of measurement. When compared to RSSI/ToA ranging and AoA, all of which involve two nodes for each measurement, it is more difficult for interferometric ranging to propagate the location information from the beacons since four nodes are involved. To eliminate the multihop propagation issue, a simple genetic algorithm approach was taken in [16], which propagates all interferometric readings within the network to a centralized location and runs a genetic algorithm to find the node locations that match the readings. Such approach is more of theoretical interest than practical use, since any centralized method is not scalable to large networks. A Pair-wise Distance method was proposed in [30] that uses both interferometric and RSSI ranging. The method uses the interferometric ranging to derive pair-wise distances among the nodes. The node locations can then be optimized using the least square error method from the pair-wise distances. The algorithm then repetitively applies the RSSI ranging measurements to fine-tune the location estimates. Compared to the genetic algorithm, the Pair-wise Distance method is able to converge much faster. However, it is currently still a centralized algorithm, which presents the same scalability issue as the genetic algorithm.

Both of the above algorithms try to optimize for a global solution given an entire set of interferometric measurements. Intuitively, finding a global solution to the localization problem is often difficult because of the large search space and the large number of constraints given by the interferometric measurements. Thus, it is desirable to find the solutions in some subspaces first and then incrementally build up to the global solution. For instance, an iterative approach has been proposed in [31] that localizes from a 
small set of seeding beacons. As additional nodes have been localized at each round, they act as pseudo-beacons that allow other nodes to be localized at subsequent rounds. The iterative method is fully distributed. However, error propagation can be an issue since any localization error at pseudobeacons would adversely affect the localization result at subsequent rounds. Simulation results in [31] have shown a linear increase of localization error at each round.

\subsection{Hybrid Algorithms}

A combination of the above techniques can be employed to form hybrid methods. For instance, a hybrid method is proposed in [32] that uses both DV-Distance [23] and Multi-Dimensional Scaling (MDS) [49]. The algorithm contains three phases. In the first phase, a small subset of nodes are selected as reference nodes. In the subsequent phase, the reference nodes are then localized in relative coordinates using MDS. The final phase uses DV-Distance to localize the rest of the nodes in absolute coordinates. The rational behind such hybrid algorithms is to exploit the tradeoff between different localization algorithms. For example, MDS gives good localization accuracy, but as the network size is increased MDS can be costly. Meanwhile, DV-Distance is less costly, but it only works well when beacon ratio is high. With hybrid algorithms, the cost is minimized by only running MDS on the reference nodes, and the reference nodes are used as beacons for DV-Distance.

\subsection{Localization Using Mobility}

While most previous methods assume stationary beacon locations, an alternative method is to localize devices using a mobile beacon. In this method, a mobile beacon travels through the deployment area while broadcasting its location along the way. Devices localize themselves by monitoring information coming from the beacon. A straight-forward technique using the above method is described in [46], where devices are required to receive at least three communications with the same RSSI reading from the beacon. Given that the same RSSI readings imply similar distances to the beacon locations, the 
physical device location can be derived using simple geometric functions. Computation-wise, this method is simple making it suitable for resource-limited sensors. However, it requires the beacon to directly pass by the ranging area of the device. In addition, in most cases, the beacon has to pass by the device twice because the sampling positions of the beacon when the three RSSI readings are taken should not be on the same line. This method also assumes that errors are insignificant in the RSSI to distance translation.

Instead of computing the location directly, a probabilistic approach may be taken; here device location is viewed as a probability distribution over the deployment area. In [45], devices measure a series of RSSI readings from the mobile beacons and localize themselves by a sequential update process to the probability distributions of their locations. Each device starts with a uniform distribution covering the entire deployment area. As the beacon passes through, the distribution is updated to fit the received RSSI readings (using a propagation model). The method is further improved in [47] by adding the negative information (that is, the information that the beacon is out of range) as well as RSSI readings from the neighbors. These probabilistic methods provide with much improved location estimates, but have the drawback of being complex. For a deployment grid of $n$ by $n$ units, the time and space complexity is $O\left(n^{2}\right)$. As the devices such as sensors at present time have very limited resources it is difficult to implement these methods directly for the large deployment. Indeed, the experimental results shown in [45] are performed on pocket PCs, which are much more powerful than common devices such as cheap sensors.

A similar method of localizing the networks using a mobile beacon is presented in [48]. Instead of the actual probability distribution, the possible device locations are represented with a bounding box. As the beacon passes by, the area contained by the bounding box is progressively reduced as positive and negative information are processed. The bounding box method drastically simplifies the probability computation, making it possible to implement this method on devices. However, such large simplification has its side-effects in that it sacrifices the preciseness of the distribution for its simplicity as it is not possible to describe multiple possible locations with a single box. There is also an additional 
problem when noise from the ranging devices is considered. This method may work well when ranging error is minimal, however when noise is present (which is inevitable when using RSSI ranging), there might be situations where no bounding box exists to satisfy all readings.

In summary, different measurement types and their unique properties to a large degree dictate the design of localization algorithms. For instance, connectivity-based measurements can only provide coarse localization without a higher beacon percentage or nodal degrees. Range and AoA-based measurements can provide much finer localization results but they are more prone to measurement error. A quantative comparison between the more well-known algorithms such as DV-Hop, Euclidean and multilateralization can be obtained from [33], in which the comparison is done in the context of specific constraints of sensor networks, such as error tolerance and energy efficiency. Their results indicate that there is no single algorithm that performs "best" and that there is room of further improvement.

\section{Theoretic Results}

While there have been many localization algorithms proposed for various scenarios, it is only recent that the theoretic aspects of the localization problem have been explored. We are particularly interested in the following three theoretic problems with regard to localization: i) localizablity, ii) complexity of localization, and iii) error bound.

First of all, we would like to know that given a network scenario (i.e., the nodes and their relative measurements such as ranging and angling) whether it is theoretically possible to uniquely localize the network. Such knowledge of localizablity is important to us because if we can easily identify that the scenario is impossible to localize uniquely, then it would be pointless to run any localization algorithm on it. Instead, we would have to request additional nodes or measurement data to be available (by possibly deploying more nodes or beacons) so that the localizablity requirement is satisfied. The following theorem gives the necessary and sufficient condition for distance-constrained network localizability in 
two dimensions.

Theorem 4.1. The network is localizable in two dimensions if and only if the network graph is redundantly rigid and triconnected [36, 35].

Theorem 4.1 holds only for two dimensions. The sufficient condition for higher dimension is currently unknown. To test the localizability, there exists a polynomial time algorithm $\left(O\left(n^{2}\right)\right.$, where $n$ is the number of vertices) that tests for the first-order rigidity (see [36] for one implementation). However, it is a known NP-Complete problem to test for the second-order rigidity of a graph [37]. A related but even more difficult problem is the node localizability, which asks if a particular node (instead of the entire network) is localizable. No sufficient condition of node localizability is currently known even in the two dimensional case, and thus no deterministic algorithm currently exists.

A second problem asks for the theoretic complexity of localization itself. In particular, we would like to know that given a network scenario that satisfies localizablity whether there exists a deterministic polynomial time algorithm that would localize the network. This problem deals with the NP-Completeness of localization. Unfortunately, the hardness of graph realization has been shown as NP-Hard under the measurement of distance [38], angle [40], connectivity [39, 41], and interferometric ranging [31].

The above theoretical results indicate the general intractability of the localization problem even in the ideal case where measurements (such as edge distances) are 100\% accurate. Unfortunately, measurements in the real world are a far-cry from being accurate, and any optimization method has to deal with not only different measurement types but also noise. The localization inaccuracy attributed to the measurement types and noise can be mathematically qualified using Cramer-Rao Bounds (CRB) [13]. The CRB is a lower bound on the covariance of any unbiased location estimator that uses measurements such as RSSI, ToA, or AoA. Thus, the CRB indicates a lower bound of the estimation accuracy of a given network scenario regardless of the localization algorithm. In other words, with CRB we have a way to tell the best any localization algorithm can do given a particular network, measurement type and 
measurement noise scenario. CRB formulas of individual measurement types such as RSSI, ToA and AoA under most common noise models (mostly Gaussian) are currently known.

The CRB of the localization error for a sample network is shown in Figure 8 as rings of radius $=\sigma_{i}$. Here, the square nodes are the beacons and the circle nodes are to be localized using RSSI ranging. The edges indicate the communication links available to measure RSSI readings. We assume the measurement model to be RSSI with the path loss exponent $n_{p}=2$ and the standard deviation of the noise $\sigma_{d B}=0.7$. A ring with smaller radius (i.e., a smaller CRB) signals that more accurate localization result can be theoretically obtained. Conversely, a larger ring indicates a larger localization variance and thus a less accurate result. In the figure, two types of nodes do not have rings. First, all beacons have a $\mathrm{CRB}$ of 0 . There are also regular nodes that have infinite $\mathrm{CRB}$ indicating that those nodes are theoretically impossible to localize. The latter case can be seen at nodes 38, 48, 49 and 78 in the top left corner. At the minimum, three beacons are needed to localize a connected network. However, those nodes in the top left corner are isolated to a different partition. Since they are connected to only one beacon (node 91), those nodes clearly cannot be localized. Other than those cases, the CRB rings at the main network partition clearly show the level of localization difficulty under various scenarios. In general, we observe that nodes closer to the beacons tend to have a smaller CRB than the ones that are several hops away. Even smaller CRB can be obtained when a node is closer to more than one beacon. All of the above observations match our common intuition about localization difficulty.

It is important to note that CRB is essentially a theoretic bound that depends on the measurement model. In the real world, its usefulness is limited by how accurate the measurement model reflects the reality. Nevertheless, CRB can be a useful tool in comparing various localization algorithms. It can be used to validate how close a particular algorithm can come to this theoretic lower bound and to see if there is any room for improvement in the algorithm design. 


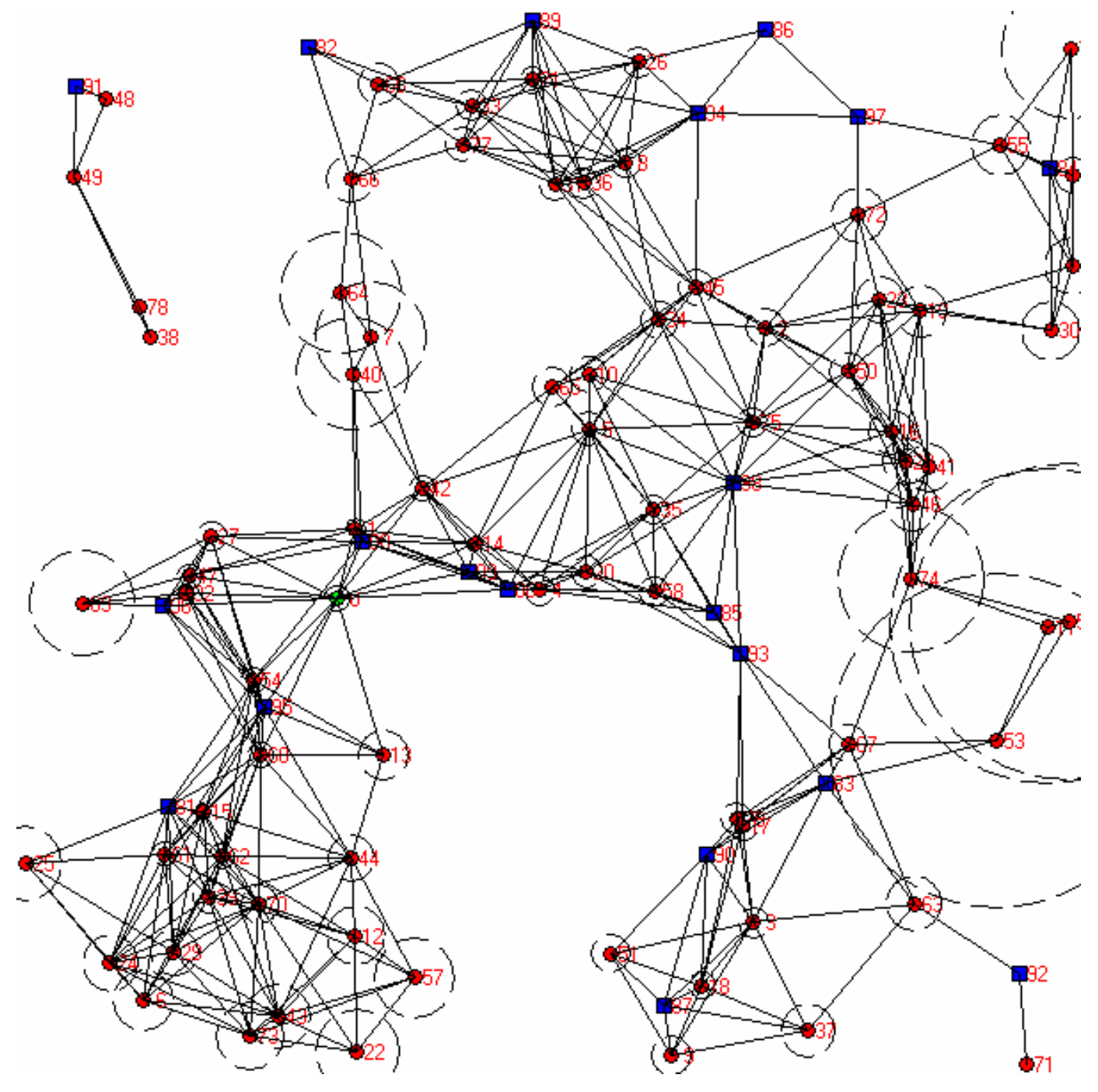

Figure 8: The CRB of the sample network is depicted as rings of the radius $=\sigma_{i}$. There are two exceptions: 1) beacons, depicted as squares, have $0 \mathrm{CRB}$, and 2) some regular nodes have infinite CRB (such as node 38, 48, 49 and 78 at the top left corner) indicating that they cannot be localized 


\section{Future Directions}

Device localization within ubiquitous computing environment has been an active research field in the past several years. Much work has been done in the area of hardware design (in particular, reducing the form factor and power consumption of sensory devices), algorithmic design and theoretical analysis. However, like many areas of ubiquitous computing, localization is still a relatively new front with much of the work yet to be done. In this section, we will briefly discuss a few directions which we feel that would produce fruitful results in the near future on the localization problem. We hope our discussion would serve the purpose to motivate the readers to actively participate and contribute their own ideas to this field.

\subsection{Implementation and Testing Environment}

When reviewing the previous works on localization, one cannot help but noticing a disturbing trend: a majority of works on localization have been based on either theoretical models or simulations, while works based on the result of actual hardware implementation have been relatively few. It is not difficult to project that the primary reason for such trend is the hardware cost. To perform meaningful experiments for localization, especially for those collaborative localization methods such as DV-Distance and Euclidean, one would normally need a large number (100+) of devices. Although mobile ad hoc network devices (for instance, sensors) are becoming cheaper by the day, it is still quite costly to implement them on physical devices in such large scale. In addition, the sensing capacities of the current devices are usually limited to RSSI. Currently, there is no cheap hardware that implements AoA, ToA, or interferometric ranging, and thus most works using those measurement types are all based on simulations. In a sense, the advances in algorithmic work on the localization problem are currently outgrowing the advances in hardware. Future work needs to be done to significantly improve the hardware design to fill this gap.

Another issue related to the testing environment is that there is no common localization testbed. 
While large scale network simulators such as NS2 [42] has a module for simulating mobile ad hoc networks, the module does not contain localization. Smaller simulators for sensor networks such as SENSE [43] usually lack features on localization as well. Another simulator SENS [44] does explicitly implement localization, but it uses a rather primitive Centroid method and lacks more sophisticated methods. Since NS2 is the most widely-used network simulator, it would be very helpful for researchers to implement an interface to NS2 that allows a "plug-in" for future localization algorithms. This would give a common testbed for different localization schemes. More importantly, it would also allow those location-depended algorithms (such as location-aided routing methods) to be implemented and compared based on the result of localization. The localization module should also implement the Cramer Rao Bounds(CRB) so that the theoretic error bound can be calculated for different localization scenarios.

\subsection{Interferometric Ranging}

Since interferometric ranging is a relatively new type of measurement available to the localization problem, there are still many open problems in this area. Of the localization algorithms proposed for interferometric ranging, all but the iterative algorithm proposed in [31] are centralized. There is a definite need to design distributed localization algorithms for interferometric ranging so that it can be implemented with reasonable efficiency and scalability. To reduce the number of beacons, the distributed algorithms should make use of multi-hop location information, which unfortunately is much more difficult for interferometric ranging because each measurement involves four nodes. For instance, there is a scheduling issue, both at a high level and low level, of when a device should be scheduled to send or receive interferometric readings. At a high level, since not all q-ranges are independent, it is more desirable to schedule the senders and receivers in order to generate more independent q-ranges instead of dependent ones. At the low level, after the senders and receivers are selected, they have to be scheduled to coordinate the signal transmission because the transmission needs to be synchronized. 
The design of the scheduling algorithm can have a substantial impact on the overall performance of the localization algorithm, in terms of the localization accuracy and communication overhead.

Furthermore, simulation study in [31] has shown that the multi-hop error propagation has a big impact on interferometric ranging, which increases almost linearly as the localization results are propagated at each hop. Therefore, the control of the error propagation is another issue. There is also a need for an algorithmic independent theoretic error bound (like CRB) for interferometric ranging. The bound would be more difficult to derive than those for distance ranging and angling because more than two nodes are involved in each measurement. Thus, the inter-dependence between the error and the relative locations of senders and receivers becomes more challenging to characterize mathematically. However, the payoff of obtaining such bound is that it would allow us to ultimately compare interferometric ranging with other measurement types and identify the scenarios that are preferable for each measurement type.

\subsection{Collaborative Localization of Multiple Measurement Types}

Previous localization algorithms often assume that the entire networks are to be localized using the same type of measurement (such as connectivity-only, RSSI, ToA, AoA, or interferometric ranging). However, to be true to the spirit of ubiquitous computing, it is foreseeable that future networks will be consisted of devices of vastly different capacities in terms of i) different transmission coverage, ii) power requirement, and iii) measurement sensors. Thus, during localization it is often desirable to explicitly consider varied devices capacities in such heterogeneous networks. For instance, in terms of varied power requirement, the localization algorithm should exploit the devices with more power capacity and try to minimize the calculation performed on less powerful devices. Furthermore, different measurement types have different error characteristics. It would be interesting to investigate how to collaborate multiple measurement types during localization, and in particular how the collaboration would impact the localization error. Intuitively, incorporating multiple measurement types in the same 
localization scenario should improve performance since such collaboration can potentially cover the drawbacks of individual measurement types for each other. Unfortunately, such collaboration could mean an increased complexity of the localization algorithms since heterogeneous networks would invalidate some assumptions often made by simple localization algorithms (such as uniform transmission range). Thus, while it is worthwhile to consider collaborative localization algorithms, it is equally imperative to keep the localization overhead under control.

\section{Summary}

In this chapter, we studied the localization problem in ubiquitous computing environment. Localization in general refer to the problem of identifying the physical location of the devices from a limited amount available measurement data. The most common measurement types include device connectivity (i.e., whether two devices are neighbors), ranging using RSSI and ToA, angle of arrival (AoA) and interferometric ranging. Given a small number of nodes with accurate geometric location using GPS receivers, localization algorithms try to derive the location of those devices that are not GPS-enabled. The motivation of localization can be justified by the large number of algorithms proposed for ubiquitous computing that rely on (semi-)accurate location information and the fact that current technology prevents GPS to be installed on all network devices due to power constraints and form factors. It has been shown that localization in general, regardless of the measurement types used, is an NP-Hard problem. Thus, current effort in solving it relies on some sort of stochastic optimization. Meanwhile, as with other network-related problems in ubiquitous computing environment, the ideal solution calls for a distributed but efficient implementation, which leads to additional challenges.

Like other aspects of ubiquitous computing, the localization problem is relatively new. The problem is also conceptually straight-forward to characterize, and many results from other disciplines such as graph theory, optimization theory, online algorithms can be readily applied to this problem. Thus, researchers from other area can provide valuable insight that could lead to better solutions to the prob- 
lem. It is our hope that this brief introduction would provide the readers motivations and inspirations to research in this field.

\section{References}

[1] Y. Ko and N. H. Vaidya. "Location-Aided Routing (LAR) in Mobile Ad Hoc Networks," Wireless Networks, vol. 6(4), pp. 307-321, July, 2000.

[2] Y. Ko and N. Vaidya. "Geocasting in Mobile Ad Hoc Networks: Location-Based Multicast Algorithms," in Proc. of IEEE WMCSA'99, pp. 101, 1999.

[3] W.-H. Liao, Y.-C. Tseng, and J.-P. Sheu. "GRID: A Fully Location-Aware Routing Protocol for Mobile Ad Hoc Networks," Telecommunication Systems, vol. 18(1), pp. 37-60, 2001.

[4] W.-H. Liao, Y.-C. Tseng, K.-L. Lo, and J.-P. Sheu. "Geogrid: A Geocasting Protocol for Mobile Ad Hoc Networks Based on Grid," Journal of Internet Technology, vol. 1(2), pp. 23-32, 2000.

[5] F. Kuhn, R. Wattenhofer, Y. Zhang, and A. Zollinger. "Geometric Ad-Hoc Routing: Of Theory and Practice," in Proc. of ACM PODC'03, pp. 63-72, 2003.

[6] X. Jiang and T. Camp. "Review of Geocasting Protocols for a Mobile Ad Hoc Network," in Proc. of the Grace Hopper Celebration (GHC), 2002.

[7] M. Mauve, H. Fuler, J. Widmer, and T. Lang. "Position-Based Multicast Routing for Mobile AdHoc Networks," Technical Report TR-03-004, Department of Computer Science, University of Mannheim, 2003.

[8] Y. Xu, J. Heidemann, and D. Estrin. "Geography-Informed Energy Conservation for Adhoc Routing," in Proc. of ACM/IEEE MOBICOM'01, pp. 70-84, 2001.

[9] Y.-C. Hu, A, Perrig, and D. Johnson. "Packet Leashes: A Defense against Wormhole Attacks in Wireless Ad Hoc Networks," in Proc. of INFOCOM'03, vol. 3, pp. 1976-1986, April 2003. 
[10] I. Stojmenovic, A. P. Ruhil, and D. K. Lobiyal. "Voronoi Diagram and Convex Hull Based Geocasting and Routing in Wireless Networks," Wireless Communications and Mobile Computing, vol. 6(2), pp. 247-258, March 2006.

[11] N. Patwari, A. O. Hero III, M. Perkins, N. S. Correal, and Robert J. O’Dea. "Relative Location Estimation in Wireless Sensor Networks," IEEE Transactions on Signal Processing, vol. 51(8), pp. 2137-2148, August 2003.

[12] B. Parkinson et al. "Global Positioning System: Theory and Application," Volume I, Progress in Astronautics and Aeronautics, vol. 163, 1996.

[13] N. Patwari, A. Hero, J. Ash, R. Moses, S. Kyperountas, and N. Correal. "Locating the Nodes: Cooperative Geolocation of Wireless Sensors," IEEE Signal Processing Magazine, vol. 22(4), pp. 54-69, July 2005.

[14] J.N. Ash and L.C. Potter. "Sensor Network Localization via Received Signal Strength Measurements with Directional Antennas," in Proc. of 2004 Allerton Conf. Communication, Control, and Computing, pp. 1861-1870, 2004.

[15] B. Kusý, M. Maróti, G. Balogh, P. V olgyesi, J. Sallai, A. Nádas, A. Lédeczi, and L. Meertens. "Node Density Independent Localization," in Proc. of 5th International Conference on Information Processing in Sensor Networks (IPSN 2006), pp. 441-448, April 2006.

[16] M. Maróti, B. Kusý, G. Balogh, P. V olgyesi, A. Nádas, K. Molnár, S. Dóra, and A. Lédeczi. "Radio Interferometric Geolocation," in Proc. of ACM 3rd Conference on Embedded Networked Sensor Systems (SenSys), pp. 1-12, Nov. 2005.

[17] F. Sivrikaya and B. Yener. "Time Synchronization in Sensor Networks: A survey," IEEE Network, vol. 18(4), pp. 45-50, Jul-Aug. 2004.

[18] N. Priyantha, A. Miu, H. Balakrishnan, and S. Teller. "The Cricket Compass for Context-Aware Mobile Applications," in Proc. of 6th ACM MOBICOM, pp. 1-14, Italy, 2001. 
[19] D. Niculescu and B. Nath. "VOR Base Stations for Indoor 802.11 Positioning," in Proc. of 10th Annual International Conference on Mobile Computing and Networking, pp. 58-69, Philadelphia, PA, USA, 2004.

[20] K. Chintalapudi, R. Govindan, G. Sukhatme, and A. Dhariwal. "Ad-Hoc Localization Using Ranging and Sectoring,' in Proc. of IEEE INFOCOM '04, pp. 2662-2672, April 2004.

[21] N. Bulusu, J. Heidemann, and D. Estrin. "GPS-less Low Cost Outdoor Localization for Very Small Devices," IEEE Personal Communications Magazine, vol. 7/5, pp. 28-34, October 2000.

[22] T. He, C. Huang, B. M. Blum, J. A. Stankovic, and T. F. Abdelzaher. "Range-Free Localization Schemes in Large Scale Sensor Networks," in Proc. of ACM MOBICOM'03, pp. 81-95, 2003.

[23] D. Niculescu and B. Nath. "Ad Hoc Positioning System (APS)," in Proc. of IEEE GLOBECOM’01, vol. 5, pp. 2926-2931, San Antonio, 2001.

[24] A. Savvides, C.-C. Han, and M. Srivastava. "Dynamic Finegrained Localization in Ad-Hoc Networks of Sensors," in Proc. of 7th ACM Int. Conf. on Mobile Computing and Networking (Mobicom), pp. 166-79, Rome, Italy, July 2001.

[25] C. Savarese, J. Rabay, and K. Langendoen. "Robust Positioning Algorithms for Distributed AdHoc Wireless Sensor Networks," in Proc. of USENIX Technical Annual Conference, pp. 317-327, Monterey, CA, June 2002.

[26] R. Huang and G. V. Záruba. "Location Tracking in Mobile Ad Hoc Networks Using Particle Filters," in Proc. of ADHOC-NOW'05, pp. 85-98, 2005.

[27] L. Hu and D. Evans. "Localization for Mobile Sensor Networks," in Proc. of ACM MOBICOM'04, pp. 45-57, 2004.

[28] A. Savvides, H. Park, and M. B. Srivastava. "The n-Hop Multilateration Primitive for Node Localization Problems," Mobile Networks and Applications, vol. 8, pp. 443-451, 2003.

[29] D. Niculescu and B. Nath. "Ad Hoc Positioning System (APS) Using AoA,” in Proc. of IEEE INFOCOM’03, vol. 3, pp. 1734-1743, San Francisco, 2003. 
[30] N. Patwari and A. O. Hero,"Indirect Radio Interferometric Localization via Pairwise Distances," in Proc. of 3rd IEEE Workshop on Embedded Networked Sensors (EmNets 2006), pp. 26-30, Boston, MA, May 2006.

[31] R. Huang and G. V. Záruba. "Complexity and Error Propagation of Localization Using Interferometric Ranging," submitted for publication, 2006.

[32] A. A. Ahmed, H. Shi, and Y. Shang. "SHARP: A New Approach to Relative Localization in Wireless Sensor Networks," in Proc. of 25th IEEE International Conference on Distributed Computing Systems Workshops (ICDCS 2005), pp. 892-898, June 2005.

[33] K. Langendoen and N. Reijers. "Distributed Localization in Wireless Sensor Networks: a Quantitative Comparison," Computer Networks, vol 43(4), pp. 499-518, Nov. 2003.

[34] J. Aspnes, T. Eren, D. K. Goldenberg, A. S. Morse, W. Whiteley, Y. R. Yang, B. D. O. Anderson, and P. N. Belhumeur. "A Theory of Network Localization," to appear in IEEE Transactions on Mobile Computing.

[35] A. Berg and T. Jordan. “A Proof of Connelly's Conjecture on 3-connected generic Cycles," Journal of Combinatorial Theory Series B, vol. 88(1), pp. 77-97, May 2003.

[36] B. Hendrickson. “Conditions for Unique Graph Realizations,” SIAM Journal on Computing, vol. 21(1), pp. 65-84, 1992.

[37] J. Saxe. "Embeddability of Weighted Graphs in k-space is Strongly NP-hard," in Proc. of 17th Allerton Conference in Communications, Control and Computing, pp. 480-489, 1979.

[38] T. Eren, D. Goldenberg, W. Whiteley, Y. R. Yang, A. S. Morse, B. D. O. Anderson, and P. N. Belhumeur. "Rigidity, Computation, and Randomization of Network Localization," in Proc. of IEEE INFOCOM'04, vol. 4, pp. 2673-2684, Hong Kong, China, Apr. 2004.

[39] H. Breu and D. G. Kirkpatrick. "Unit Disk Graph Recognition is NP-hard," Computational Geometry. Theory and Applications, vol. 9(1-2), pp. 3-24, 1998. 
[40] J. Bruck, J. Gao, and A. Jiang. "Localization and Routing in Sensor Networks by Local Angle Information," in Proc. of 6th ACM International Symposium on Mobile Ad Hoc Networking and Computing, pp. 181-192, Urbana-Champaign, IL, USA, 2005.

[41] F. Kuhn, T. Moscibroda, and R. Wattenhofer. “Unit Disk Graph Approximation,” in Proc. of Workshop on Discrete Algorithms and Methods for Mobile Computing and Communications (DIAL-M), pp. 17-23, Philadelphia, PA, USA, 2004.

[42] The Network Simulator - ns-2, Internet Resource. http://www.isi.edu/nsnam/ns/.

[43] G. Chen, J. Branch, M. J. Pflug, L. Zhu, and B. Szymanski. "SENSE: A Sensor Network Simulator," Advances in Pervasive Computing and Networking, pp. 249-267, Springer, 2004.

[44] S. Sundresh, W. Kim, and G. Agha. "SENS: A Sensor, Environment and Network Simulator," in Proc. of 37th Annual Simulation Symposium (ANSS37), Arlington, VA, April, 2004.

[45] M. L. Sichitiu and V. Ramadurai. "Localization of Wireless Sensor Networks with a Mobile Beacon," in Proc. of 1st IEEE Conference on Mobile Ad-hoc and Sensor Systems (MASS 2004), pp. 174-183, Fort Lauderdale, FL, October 2004.

[46] K.-F. Ssu, C.-H. Ou, and H.C. Jiau. "Localization with Mobile Anchor Points in Wireless Sensor Networks," IEEE Vehicular Technology Conference (VTC 2005), vol. 54(3), pp. 1187-1197, May 2005

[47] R. Peng and M. L. Sichitiu. "Robust, Probabilistic, Constraint-Based Localization for Wireless Sensor Networks," in Proc. of 2nd Annual IEEE Communications Society Conference on Sensor and Ad Hoc Communications and Networks (SECON 2005), pp. 541-550, Santa Clara, CA, Sep. 2005.

[48] A. Galstyan, B. Krishnamachari, K. Lerman, and S. Pattem. "Distributed Online Localization in Sensor Networks Using a Moving Target,” in Proc. of IPSN 2004, pp. 61-70, April 2004.

[49] Y. Shang, W. Ruml, Y. Zhang, and M. Fromherz. "Localization from Mere Connectivity," in Proc. of ACM MobiHoc’03, pp. 201-212, Annapolis, MD, June 2003. 